\title{
Bandwidth-limited picosecond pulse generation in an actively mode-locked GaAs laser with intracavity chirp compensation
}

\author{
J. Kuhl and M. Serenyi* \\ Max-Planck-Institut für Festkörperforschung, 7000 Stuttgart 80, Federal Republic of Germany
}

E. O. Göbel

Fachbereich Physik, Philipps University, 3550 Marburg, Federal Republic of Germany

Received January 5, 1987; accepted February 10, 1987

A Gires-Tournois interferometer has been applied for intracavity chirp compensation of an actively mode-locked

GaAs laser. Fourier-transform-limited pulses as short as 4.6 psec have been obtained at $790 \mathrm{~nm}$.

\begin{abstract}
Mode locking of semiconductor lasers in an external cavity is an attractive technique for the generation of picosecond optical pulses for optical-communication and data-processing systems. Active mode locking is accomplished by microwave current or pulse current injection if the frequency is matched to the photon round-trip time within the external resonator. Typical values for the pulse width in the range of 5.3-30 psec have been reported. ${ }^{1-5}$ In addition to the pulse width, the spectral width of the mode-locked pulses is of importance if, e.g., application in coherent-opticalcommunication systems is considered. The characterization of a mode-locked pulse therefore requires simultaneous analysis of the temporal and spectral shapes of the emission. If the pulses are totally coherent, which provides the minimum spectral width for a given pulse width, the time-bandwidth product varies between 0.11 and $0.44,{ }^{6}$ depending on the pulse profile. Many efforts to reduce the pulse duration of modelocked semiconductor lasers reveal the difficulties in approaching the Fourier-transform limit. This is partly because the spectrum often exhibits a distinct substructure reflecting the gain modulation by the Fabry-Perot interference pattern of the short-diode crystal. ${ }^{7}$ The pulse duration attainable from such a modulated spectrum is limited by the width of the individual Fabry-Perot mode, according to the rules of Fourier transformation. The excessive bandwidth generates only substructure below the pulse envelope but cannot be utilized for pulse shortening. Suppression of the mode structure caused by the short crystal can be accomplished by antireflection (AR) coatings, ${ }^{8}$ by insertion of narrow-band étalons into the external resonator, ${ }^{4}$ or by fabrication of diodes with the active stripe tilted with respect to the crystal faces. ${ }^{2}$

In addition to this unwanted multimode structure, diode lasers emitting pulses in the picosecond time regime usually exhibit spectral broadening of the modes comparable with the longitudinal mode spacing of the semiconductor crystal. This dynamic broadening (chirp) is explained by the carrier-density dependence of the refractive index. ${ }^{9,10}$
\end{abstract}

If the laser is operating close to the gain maximum, the refractive-index change associated with the emission of a pulse is dominated by the decrease of the carrier concentration, causing an increase of the refractive index and hence a red shift of the emission (downchirp).

The recent progress in the generation of optical pulses well below 100 -fsec duration from collidingpulse mode-locked ring dye lasers has demonstrated the potential of optical devices with tunable groupvelocity dispersion for pulse compression. By employing intracavity frequency-chirp compensation, pulses as short as 27 fsec have been realized. ${ }^{11}$

In this Letter we report on intracavity chirp compensation of an actively mode-locked GaAs/AlGaAs laser using thin Gires-Tournois interferometers (GTI's). We showed in a previous paper ${ }^{12}$ that the pulse duration of a mode-locked GaAs laser can be considerably reduced if the emission wavelength is tuned from the gain maximum to higher photon energies. Combining operation at the short-wavelength edge of the emission spectrum and chirp compensation, we have now succeeded in generating pulses as short as $4.6 \mathrm{psec}$ at $790 \mathrm{~nm}$. The time-bandwidth product of 0.13 is very close to the Fourier-transform limit for pulses with a single-sided exponential profile.

The experimental setup, depicted in Fig. 1, is based on the conventional configuration for active mode locking of semiconductor lasers. Many details have already been described in Ref. 12 . The diode is an index-guided buried-heterostructure laser (Hitachi 3400 ) temperature stabilized to $20^{\circ} \mathrm{C}$. For the present experiments the external optical resonator includes the GTI $^{13}$ and an additional $100 \%$ mirror adjusted parallel to each other and mounted on a common turntable for angle tuning of the group-velocity dispersion and an interference filter for wavelength tuning.

The interferometer is fabricated by covering the front and back surfaces of a $300-\mu \mathrm{m}$ solid quartz étalon with dielectric mirrors of 30 and $100 \%$ reflectivity, respectively. Small angles of incidence onto the relatively thick étalon are necessary for sufficient spectral 


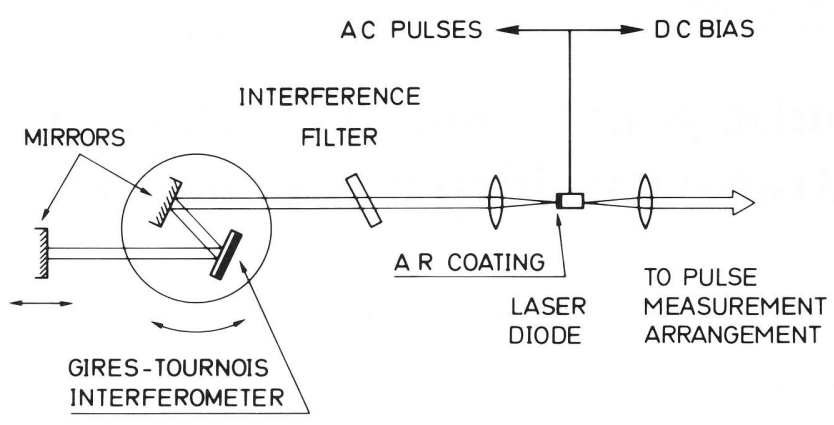

Fig. 1. Experimental setup of the diode laser cavity with internal chirp compensation.

selectivity to be achieved. The antireflection coating on the cleaved diode-laser surface facing the external cavity has a residual reflectivity of $<2 \times 10^{-4} .{ }^{14}$ The $\mathrm{cw}$ threshold of the entire laser setup amounts to 18.5 $\mathrm{mA}$ at the gain maximum $(\lambda=823 \mathrm{~nm})$ and increases to $50 \mathrm{~mA}$ if the interference filter is tuned to the shortwavelength limit. The diode is prebiased by a dc current well below threshold with short electrical pulses from a comb generator (duration 130 psec, repetition rate $80 \mathrm{MHz}$ ) superimposed in order to produce the rapid gain modulation above the threshold level.

The mode-locked laser output is spectrally analyzed by a 1 -m double-grating monochromator with a resolution of $0.1 \mathrm{~cm}^{-1}$. Depending on the pulse duration, either a synchroscan streak camera (resolution 10 psec) or autocorrelation techniques utilizing secondharmonic generation (SHG) in a $15-\mathrm{mm}$-long $\mathrm{LiIO}_{3}$ crystal have been employed for measurements of the pulse width.

Figure 2 displays the SHG autocorrelation trace together with the spectrum for the shortest pulses produced at $791 \mathrm{~nm}$. In this particular case the diode is biased with $3.5-\mathrm{mA}$ dc current with pulses of $280-\mathrm{mA}$ peak amplitude superimposed. The carrier density is estimated to be $\sim 4 \times 10^{18} \mathrm{~cm}^{-3}$ at this strong pumping level, resulting in considerable band filling, which is required for laser operation at this high photon energy. The average output power is $160 \mu \mathrm{W}$. Small cavity-length changes of $\pm 10 \mu \mathrm{m}$ from the optimum value lead to easily detectable broadening of the autocorrelation trace. Thus the sensitivity to cavity detuning is comparable with that of the synchronously pumped dye laser. ${ }^{15}$

The strikingly broad wings of the correlation function suggest the assumption of a single-sided exponential pulse profile. This assumption is confirmed by the exponential slope of the trace, as demonstrated by the logarithmic plot of SHG intensity shown in the inset. The autocorrelation trace for a single-sided exponential pulse shape (FWHM $=t_{1 / 2}$ ) is given by $I(\Delta t) \sim \exp \left[-(\ln 2) \Delta t / t_{1 / 2}\right] .{ }^{6} \quad$ Deconvolution of the autocorrelation trace in Fig. 2 thus yields a pulse duration (FWHM) of 4.6 psec. The corresponding emission spectrum of the mode-locked pulses is also shown in Fig. 2. The spectrum is appreciably narrower than the transmission band of the interference filter and is determined by the overlap of the filter transmission and (toward shorter wavelengths) the sharply decreas- ing gain profile. The spectral width of the emission is $0.06 \mathrm{~nm}$, and thus the time-bandwidth product is 0.13 , which is very close to the transform-limited value of $0.11 .^{6}$ Significant pulse shortening for increasing photon energy and a highly asymmetric pulse shape (in particular, pulses with a rise time much slower than the fall time) were observed previously at the highenergy edge of the stimulated emission from semiconductor lasers pumped by subpicosecond optical pulses. ${ }^{16}$ In addition, these authors show that the unusual pulse shape is predicted by a rate-equation model including the dynamics in the hot-electron and hole-energy distributions. Pulse shortening is a direct consequence of the short lifetime of transient gain for photon energies far above the band gap because of the rapid decrease of the Fermi level.

The influence of intracavity chirp compensation is illustrated in Fig. 3. The measured FWHM of the intensity autocorrelation trace is plotted versus the angle of incidence of the intracavity beam onto the GTI. The solid line represents the calculated groupvelocity dispersion of the interferometer, which can be varied continuously between $\pm 8 \mathrm{psec} / \mathrm{THz}$ by angle tuning. The dots correspond to measured pulse widths with the laser wavelength fixed at $790 \mathrm{~nm}$. The dashed line connects the data points for better visualization. The laser cavity length was optimized for each measurement to eliminate unavoidable variations of the cavity length due to tuning of the GTI. The shortest pulses are obtained if the GTI provides positive group-velocity dispersion, as expected for a downchirped pulse. If the dispersion is tuned to zero, an increase of the pulse duration by about $20 \%$ occurs. Negative group-velocity dispersion leads to remarkable pulse stretching.

Attempts to apply the same intracavity pulse-compression technique for shortening of pulses at the gain maximum failed. We attribute this negative result to the fact that the 20-psec pulse duration at the gain maximum is determined mainly by the long time persistence of the gain at lower photon energies. This

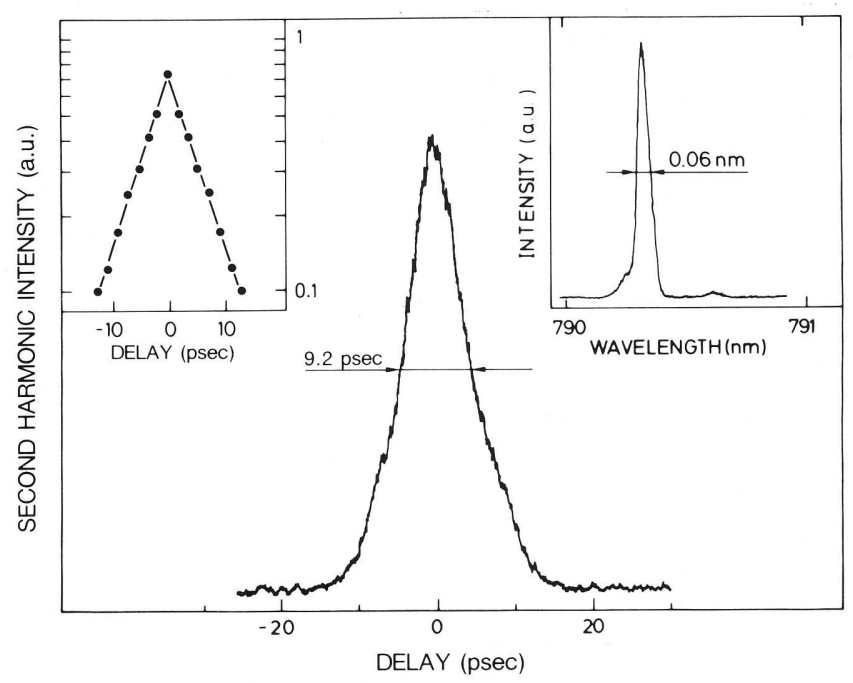

Fig. 2. Intensity autocorrelation function and spectrum of mode-locked pulses at $790 \mathrm{~nm}$. 


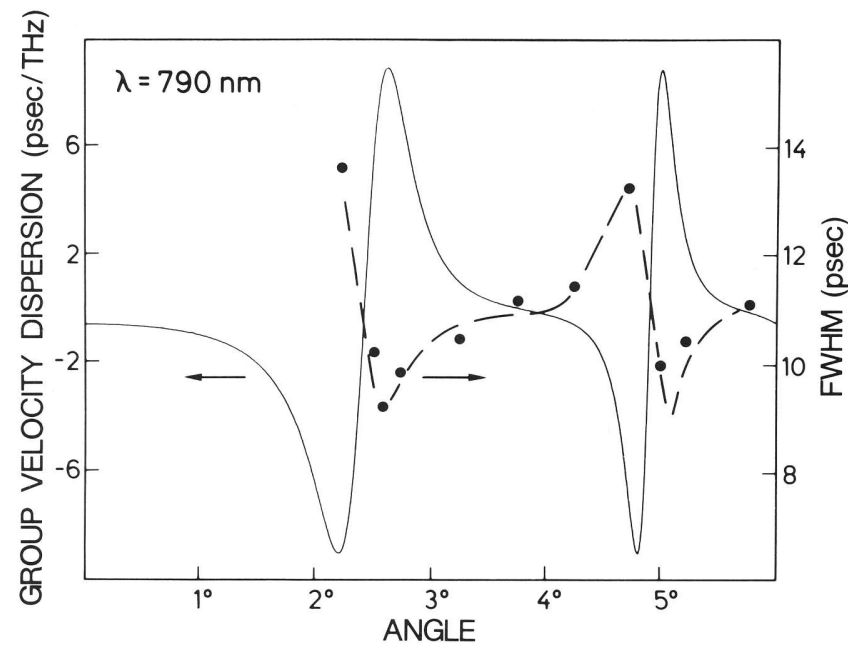

Fig. 3. Group-velocity dispersion (solid line) of the intracavity GTI and measured full width at half-maximum of the intensity autocorrelation trace (dots) versus angle of incidence.

effect is a consequence of the carrier dynamics induced by recombination heating of the electron-hole plasma during lasing. ${ }^{16}$ Compared to this process, the contributions of chirping associated with the refractive-index modulation during gain depletion seem to be of minor importance for pulse broadening at the gain maximum.

In conclusion, we have demonstrated the generation of bandwidth-limited optical pulses as short as 4.6 psec from an actively mode-locked buried-heterostructure GaAs/AlGaAs laser by tuning the wavelength to the high-energy edge of the gain spectrum and applying GTI's for intracavity chirp compensation.

Note added in proof: After submission of the manuscript of this Letter, it was brought to our attention that R. S. Putnam and M. M. Salour ${ }^{17}$ have also applied interferometers for intracavity pulse compression of mode-locked semiconductor lasers.

* On leave from the Research Institute for Technical Physics of the Hungarian Academy of Sciences, H1325 Budapest, Hungary.

\section{References}

1. J. P. van der Ziel, J. Appl. Phys. 52, 4435 (1981).

2. M. B. Holbrook, W. E. Sleat, and D. J. Bradley, Appl. Phys. Lett. 37, 59 (1980).

3. J. C. AuYeung and A. R. Johnston, Appl. Phys. Lett. 40, 112 (1982).

4. E. O. Göbel, J. Kuhl, and G. Veith, J. Appl. Phys. 56, 862 (1984).

5. J. McInerney, L. Reekie, and D. J. Bradley, Electron. Lett. 21, 117 (1985).

6. E. P. Ippen and C. V. Shank, in Ultrashort Light Pulses, S. L. Shapiro, ed. (Springer-Verlag, Berlin, 1977), p. 88.

7. D. Marcuse and T. P. Lee, IEEE J. Quantum Electron. QE-19, 1397 (1983).

8. G. Eisenstein and L. W. Stulz, Appl. Opt. 23, 161 (1984).

9. J. P. van der Ziel, IEEE J. Quantum Electron. QE-15, 1277 (1979).

10. C. Lin, T. P. Lee, and C. A. Burrus, Appl. Phys. Lett. 42, 141 (1983).

11. J. A. Valdmanis, R. L. Fork, and J. P. Gordon, Opt. Lett. 10, 131 (1985)

12. M. Serenyi, J. Kuhl, and E. O. Göbel, "Pulse shortening of actively mode-locked diode lasers by wavelength tuning," submitted to Appl. Phys. Lett.

13. J. Kuhl and J. Heppner, IEEE J. Quantum Electron. QE-22, 182 (1986); J. Heppner and J. Kuhl, Appl. Phys. Lett. 47, 453 (1985).

14. M. Serenyi and H. U. Habermeier, Appl. Opt. 26, 845 (1987)

15. C. P. Ausschnitt, R. K. Jain, and J. P. Heritage, IEEE J. Quantum Electron. QE-15, 912 (1979).

16. T. L. Koch, L. C. Chiu, Ch. Harder, and A. Yariv, Appl. Phys. Lett. 41, 6 (1982).

17. R. S. Putnam and M. M. Salour, Proc. Soc. Photo-Opt. Instrum. Eng. 439, 66 (1983). 